\title{
Patient counselling service with the use of pictograms as the example of pharmacist intervention to improving compliance and medicine safety
}

\author{
Piotr Merks ${ }^{1,2,3 *}$, Damian Świeczkowski ${ }^{4}$, Marcin Balcerzak ${ }^{5}$, Urszula Religioni ${ }^{6}$, \\ Ewelina Drelich ${ }^{2}$, Jerzy Krysiński $^{2}$, Dagmara Hering ${ }^{4}$, Miłosz Jaguszewski ${ }^{4}$ \\ ${ }^{1}$ Faculty of Medicine, Collegium Medicum, Cardinal Stefan Wyszynski University in Warsaw, Poland \\ ${ }^{2}$ Department of Pharmaceutical Technology, Collegium Medicum, Bydgoszcz, \\ Nicolaus University, Torun, Poland \\ ${ }^{3}$ Piktorex, Warsaw, Poland \\ ${ }^{4}$ First Department of Cardiology, Medical University of Gdansk, Poland \\ ${ }^{5}$ Medink, Mysiadlo, Poland \\ ${ }^{6}$ Collegium of Business Administration, Warsaw School of Economics, Warsaw, Poland
}

This paper was quest edited by Prof. Jan Saevels

\begin{abstract}
Background: Pharmaceutical pictograms have been designed to help communicate medication instructions to patients. Pictograms used within a patient counseling service can significantly improve medication compliance and adherence. The study aimed to assess the improvement of adherence to therapy with the use of pictogram intervention in comparison to standard pharmacy practice in community pharmacies.

Methods: Pictograms informing about the proper way of using metoprolol prolonged release tablets were designed to be used on the packages of the drug in community pharmacies. Pharmacies belonging to a pharmacy practice-based research network were randomly assigned to a group using pictograms when dispensing the drug or one following their normal practice. At the first visit, all patients answered a structured questionnaire about their medication behavior in the preceding 7 days. The same questions were asked 4 weeks later to follow-up treatment adherence change and compare patients from pictogram group and standard practice group. Descriptive statistics was used to analyze the data, and the $\mathrm{McNe}$ mar test was used to compare categorical data at baseline and follow-up.

Results: Of a total of 253 patients screened, 117 and 104 patients completed the study in the standard practice and pictogram groups, respectively. The use of pictograms significantly improved medication adherence in the following areas: not omitting doses $(p<0.0001)$, not crushing tablets $(p=0.004)$, number of tablets/day $(p=0.49)$, and time of use $(p=0.001)$, compared to the standard practice group. Conclusions: Our results suggest that pictograms are effective in conveying messages about the proper way of using medications, and they increase treatment adherence, in comparison to standard dispensing practice. (Cardiol J 2021; 28, 6: 879-886)
\end{abstract}

Key words: adherence, compliance, chronic disease, community pharmacy, pharmaceutical pictograms

Address for correspondence: Dr. Piotr Merks, Faculty of Medicine, Collegium Medicum, Cardinal Stefan Wyszynski University, ul. Wóycickiego 1/3, 01-938 Warszawa, Poland. tel: +48 602101979, e-mail: p.merks@uksw.edu.pl

Received: 4.01.2021 Accepted: 5.02.2021 Early publication date: 16.02.2021

This article is available in open access under Creative Common Attribution-Non-Commercial-No Derivatives 4.0 International (CC BY-NC-ND 4.0) license, allowing to download articles and share them with others as long as they credit the authors and the publisher, but without permission to change them in any way or use them commercially. 


\section{Introduction}

To date, many studies have addressed the problem of lack of adherence in patients diagnosed with chronic diseases. Due to the high prevalence and burden of cardiovascular diseases, adherence to prescribed treatment is considered among the most critical strategies contributing to improving health outcomes $[1,2]$. Of the many factors affecting medication adherence, health care providers' factors are highlighted in particular [2]. Improving communication with the patient, patients' education, and increasing patient involvement can significantly improve medication adherence. Pharmacists' counseling is especially emphasized in this area [3]. Pharmacists play a key role in handling prescriptions, and by direct contact with patients just before the use of the drug, they have great potential to encourage patient compliance [4]. Good compliance and adherence improve healthrelated outcomes in many conditions [5]. On the other hand, poor medication adherence is associated with higher mortality and a greater risk of hospitalization [6].

Pharmaceutical pictograms as a supplement to pharmacy counseling are considered an effective tool facilitating the patient's understanding of the information provided [1,7]. Pictograms are defined as visual images used in health information materials [8]. These types of drawings graphically represent instructions for the correct use and storage of medicines [7]. Pictograms enable patients to understand the instructions on how to use medicines, which is especially important in the context of health literacy. Pictograms can significantly influence the proper use of drugs among patients with low health literacy [9]. Thus, including pictograms in patient counseling could reduce the frequency of medication dosing errors. Moreover, pictograms in combination with a leaflet attached to the drug increase the patient's attention, remembering and recalling the rules of taking the drug [10]. Significantly, pictograms are culturally neutral, making them understandable regardless of language, also for people who cannot fully understand printed textual medication information [9]. Although there are many publications on pictograms in the literature, data about the utilization of pharmaceutical pictograms in a community pharmacy setting is limited. Most studies focus on design, comprehensiveness, and validation of different pictograms. High-quality studies are needed to support the routine use of any pictogram-based materials in routine practice. This study presents the results of the utilization of phar- maceutical pictograms in a community pharmacy setting. The study aimed to compare changes in adherence to treatment with metoprolol prolonged release (PR) in groups of patients who were/were not provided with pictograms when dispensing the drug at pharmacies.

\section{Methods}

\section{Design and setting}

This was a multicenter, prospective study with a control arm. The study was approved by the institutional review board of Collegium Medicum in Bydgoszcz, Poland. Patients' participation in the study was voluntary. All the patients provided written informed consent.

Patients were eligible for enrollment in the study if they were $\geq 18$ years old, had a prescription of metoprolol PR tablets, had been using the drug once daily for at least 3 months, and signed an informed consent form agreeing to follow-up contact.

Metoprolol, a beta-blocking agent, belongs to the most common drugs used in the treatment of cardiovascular diseases. Patients who had just started PR metoprolol therapy, had used immediate-release metoprolol, or had been prescribed a different dosage regimen than once daily were excluded from our study. At a 1:1 ratio between groups, levels of type I error 0.05, and type II error 0.2 the study required 93 patients per group. However, these hypothetical assumptions were not reflected in the study results.

The study was conducted in Polish community pharmacies belonging to the Farenta Research pharmacy practice-based research network. Pharmacies were randomly assigned in a 1:1 ratio to dispense the products together with 3 pictograms placed on an external package showing how to use the medicine correctly, or in line with standard pharmacy practice (control group, without any pictograms). Pharmacies $(n=50)$ were randomized to pictogram or standard practice groups using a random sequence generator from www.random. org. The study was based on 2 interviews: an initial interview conducted during dispensation and a second interview performed no more than 30 days after first one (Fig. 1). At pharmacies randomized to the pictogram group, each patient received with the drug a set of 3 pharmaceutical pictograms with the following instructions: Take 1 tablet in the morning, do not crush the tablet, and swallow the tablet with water (Fig. 2). All pictograms were validated, as described earlier [11]. The dimension of each pictogram was 


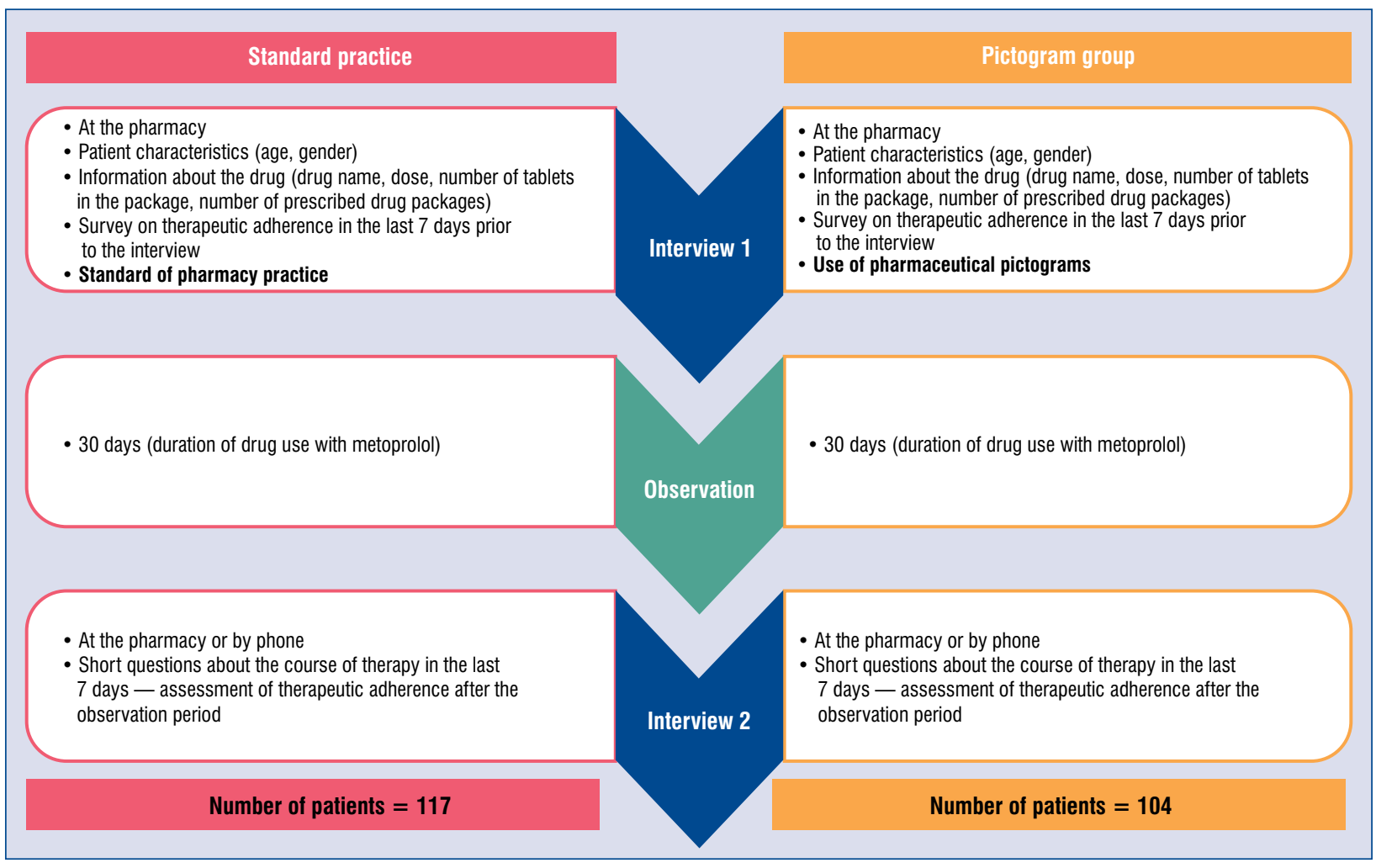

Figure 1. The use of pharmaceutical pictograms in the course of therapy with metoprolol prolonged-release tablets - the intervention chart.

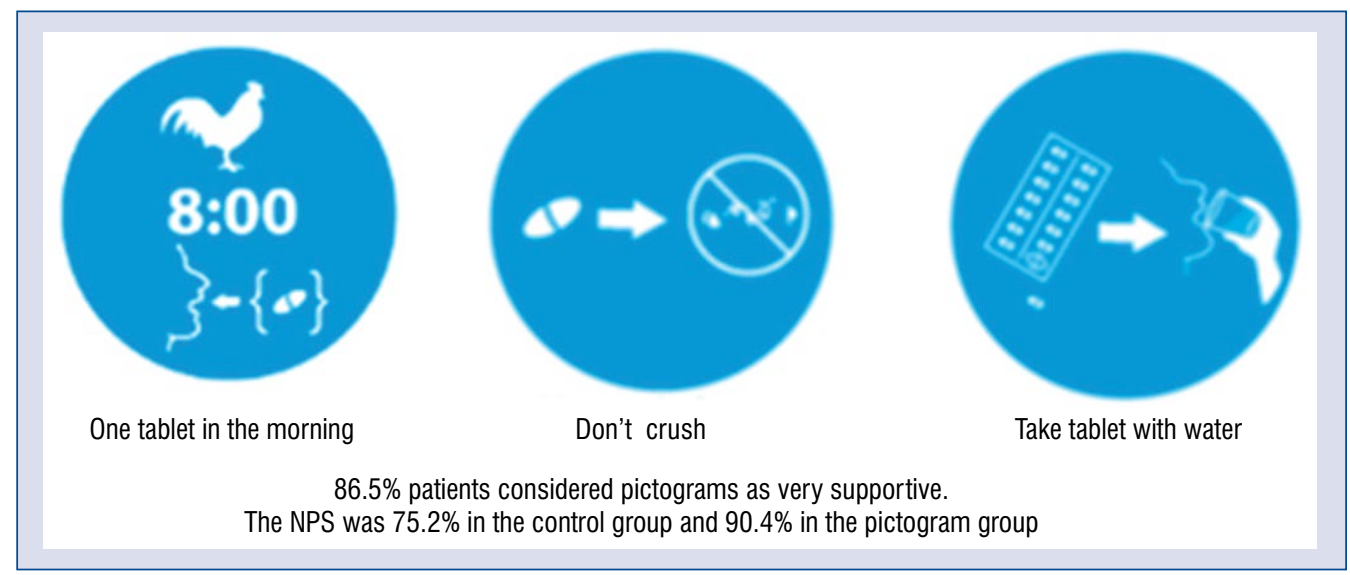

Figure 2. The set of pictograms used in the study; NPS - net promoter score.

$30 \times 30 \mathrm{~mm}$ (Fig. 2). Medical information conveyed by pictograms was consistent with the information in the local summaries of product characteristics and aligned with medication plans of patients. As recommended by the physician, the correct behavior was as follows: taking the medicine every day, taking one tablet once a day in the morning, not crushing/not chewing the tablet, swallowing the tablet with water. At pharmacies randomized to the standard practice, group patients obtained the drug without pictograms. The dispensation was ongoing according to the individual practice of pharmacists and not influenced by the study team. 


\section{Data acquisition}

The first study interview was conducted during the initial visit at the pharmacy. We gave the same questionnaires to both groups. To allow us to compare the results from both interviews, during the first interview each patient received a unique identifier. The interviews were conducted by the pharmacy employee dispensing the medicines.

The questions asked in the first interview included sociodemographic information and behavior regarding use of metoprolol PR tablets in the last 7 days. Questions were related to the following dimensions of adherence: omitting doses, time of drug use, a number of tablets taken, dosing frequency, crushing/chewing tablets, and taking them with water (these 6 dimensions are understood as full adherence). The Brief Medication Questionnaire (BMQ) inspired the design of the questions [5]. The questions were adapted to assess outcomes influenced by pictogram messages. The scoring system was similar to the BMQ. The scales with multiple choices graduating the patient's adherence behavior were used to allow patients to indicate even small deviations from the target way of the use of the drug; however, the analysis was conservative, and the only full adherence to therapy was considered as a positive outcome.

The questions used in the study and their interpretation are available in Supplementary Materials. The second interview was performed 1 month after the first visit and included the same questions as in the earlier survey about adherence behavior in the preceding 7 days. Also, patients were asked to evaluate medical information received in the community pharmacy on a scale from 1 to 10 , where $1=$ very bad and $10=$ very good. The data were recorded electronically by interviewing the employees of the participating pharmacies.

\section{Endpoints}

The primary outcome measures of the study included a change from the first to the second visit in the percentage of patients fully adherent to the main messages conveyed by the 3 pictograms: not omitting doses, not crushing tablets, swallowing tablets with water. The secondary endpoint was a change in the percentage of patients declaring the use of the correct number of individual doses during the day, the right number of tablets per dose, and taking the drug in the morning.

\section{Data analysis}

All categorical data were expressed as proportions, and continuous data were expressed as mean and standard deviation (SD). Descriptive analysis was conducted to present the results at baseline and follow up, assessing the primary and secondary outcome measures. Patients' declarations in the survey were classified as indicating full adherence (answers indicating proper behavior) or non-adherence (answers indicating any other behavior than the proper one or 'do not know'). The McNemar test was used to compare categorical data at baseline and follow-up in both groups. Intention to treat analysis was followed, and a probability value of $<0.05$ was considered statistically significant for all analyses. Possible changes from the first visit to the second visit included the following: no change in adherence, improvement, and worsening of adherence. Logistic regression was used to calculate the odds of improvement or worsening adherence in every studied dimension of adherence for the patients of each group.

The net promoter score (NPS) was used to examine the patients' opinions about medical information received at a pharmacy [12]. Patients who evaluated medical information as 9 or 10 points were classified as advocates. The group of patients who responded 7-8 were classified as indifferent, and re the respondents who evaluated the information received as 1-6 points were classified as critical of the provided information.

\section{Results}

\section{Patient disposition and characteristics}

The data were collected between January and March 2017, and in total 253 patients participated in the first interview. The number of screened patients is unknown because pharmacists did not record patients refusing to participate or ineligible to participate. The data of 32 patients were not included in the analysis because they were lost to follow-up $(n=13)$, provided incomplete data in the first interview $(n=12)$, used metoprolol immediate release $(n=2)$, were dosed differently than according to the inclusion criteria $(\mathrm{n}=3)$, or misconduct was detected $(n=2)$. The complete data from 117 patients in the standard practice group and $104 \mathrm{pa}-$ tients in the pictogram group were included in the analysis. The baseline characteristics by treatment group are presented in Table 1 . The patient sample was $59 \%$ female. Mean age \pm SD of patients was $65.2 \pm 13.0$ years. The sample included patients self-reporting use of metoprolol for hypertension (51\%), cardiac arrhythmias (29\%), prophylactic treatment following myocardial infarction (7\%), angina (5\%), and unknown/undeclared reasons (7\%). 
Table 1. Characteristics of the study population.

\begin{tabular}{lcc}
\hline & $\begin{array}{c}\text { Routine pharmacy practice } \\
(\mathbf{n}=117)\end{array}$ & $\begin{array}{c}\text { Pharmaceutical pictograms } \\
(\mathbf{n}=104)\end{array}$ \\
\hline Age, median (range) [years] & $67(31-94)$ & $64(30-91)$ \\
Woman & $74(63.2 \%)$ & $58(55.8 \%)$ \\
Different drugs used by patients: & & \\
Missing & $1(0.9 \%)$ & $0(0 \%)$ \\
1 drug & $5(4.3 \%)$ & $5(4.8 \%)$ \\
2 drugs & $15(12.8 \%)$ & $11(10.6 \%)$ \\
3 drugs & $25(21.4 \%)$ & $17(16.3 \%)$ \\
$\geq 4$ drugs & $71(60.7 \%)$ & $71(71.2 \%)$ \\
Indication for use of metoprolol: & & \\
Hypertension & $60(51.3 \%)$ & $52(50.0 \%)$ \\
Cardiac arrythmias & $40(34.2 \%)$ & $25(24.0 \%)$ \\
Prophylactic after myocardial infarction & $6(5.1 \%)$ & $10(9.6 \%)$ \\
Angina pectoris & $5(4.3 \%)$ & $7(6.7 \%)$ \\
I do not know/l do not remember & $6(5.1 \%)$ & $10(9.6 \%)$ \\
\hline
\end{tabular}

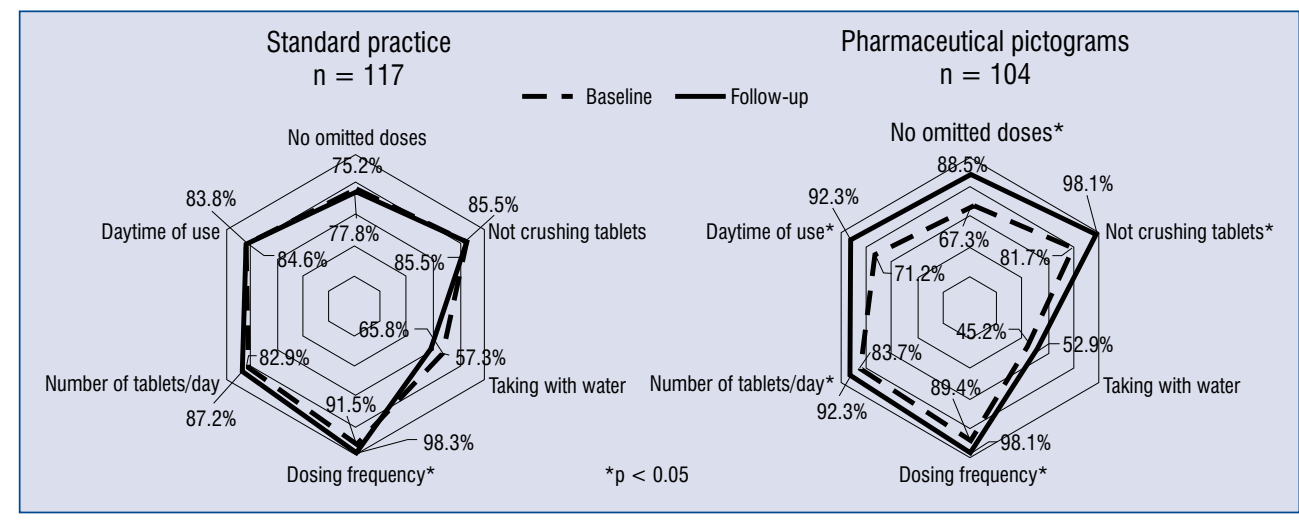

Figure 3. The percentage of patients fully adherent (proper therapeutic behavior) for each tested adherence domain in the standard practice and pictogram groups at baseline and follow-up.

\section{The practical effectiveness of pictograms}

At baseline, 74 (63.2\%) patients in the standard practice group and $70(67.3 \%)$ patients in the pharmaceutical pictograms group had $\geq 1$ problem in any of the analyzed adherence dimensions. At the follow-up, the number of non-adherent patients remained stable in the standard group $(n=76,65.0 \%)$ and decreased in the pictogram group $(\mathrm{n}=58$, $55.8 \%), p=0.1636$. Figure 3 presents changes in percentages of patients fully adherent in each of the 6 analyzed dimensions of adherence at baseline and follow-up. In the pictogram group an increase in the number of patients with full adherence was more common than in the standard practice group. The percentage of patients not omitting doses increased in the pictogram group from $67.3 \%$ to $88.5 \%$ (an increase of $31.5 \%, \mathrm{p}<0.0001$ ). In the standard practice group the percentage of patients not omitting doses remained stable (from $77.8 \%$ to $75.2 \% ; \mathrm{p}=0.8679$ ). At the follow-up, $98.1 \%$ of patients in the pictogram group were not crushing tablets, compared to $81.7 \%$ at baseline (increase by $20.07 \%, \mathrm{p}=0.004)$. In the standard practice group, the percentages of patients not crushing tablets at baseline and follow-up did not change $(85.5 \%$ vs. $85.5 \%, \mathrm{p}=0.647)$. Neither pictograms nor standard practice improved significantly for the swallowing tablets with water behavior (an increase from $45.2 \%$ to $52.9 \%$ of patients in the pictogram group, $\mathrm{p}=0.322$, and a decrease from 
$65.8 \%$ to $57.2 \%$ of patients in the standard practice group, $\mathrm{p}=0.121$; Fig. 3 ).

Pictograms improved adherence in all secondary endpoints. In the pictogram group the percentage of patients using metoprolol once daily was $89.4 \%$ at baseline and $98.1 \%$ at follow-up (an increase of $9.73 \%, p=0.004)$. However, a similar improvement was also observed in the standard practice group (an increase of $7.43 \%, \mathrm{p}=0.021$; Fig. 3). Pictograms significantly increased the number of patients using 1 tablet per day in line with a defined treatment plan from $83.6 \%$ to $92.3 \%$ (an increase of $10.27 \%, p=0.049$ ). The improvement was not significant in the standard practice group ( $p=0.359$; Fig. 3 ). Finally, pictograms increased the percentage of patients using the drug in the morning from $71.1 \%$ at baseline to $92.3 \%$ at follow-up (an increase of $29.63 \%, \mathrm{p}=0.0001$ ), but this was not the case in the standard practice group $(\mathrm{p}=1)$.

\section{Patients' opinions about pharmaceutical pictograms}

The NPS was $75.2 \%$ in the control group and $90.4 \%$ in the pictogram group. The vast majority of patients $(86.5 \% ; \mathrm{n}=90)$ who obtained pictograms identified them as very supportive. The mean score $\pm \mathrm{SD}$ in the pictogram group was $9.58 \pm 0.69$ and in the control group $9.24 \pm 1.30(\mathrm{p}=0.0129)$.

\section{Discussion}

This study provides the first comparative evidence that the use of pharmaceutical pictograms improves adherence to recommended drug therapy in routine pharmacy practice. Improvement of adherence was associated with a substantial reduction in the number of patients who missed drug doses. Importantly, pictograms have also increased awareness and knowledge about not crushing tablet medication, which has clinically relevant implications in terms of treatment effectiveness. Furthermore, patients who were using pictograms more often changed the time of taking the drug to the morning, in comparison to the standard practice. Of all these areas, only an improvement in dosing frequency was observed in the group of patients with standard practice.

The only domain of adherence that remained unchanged by pictogram use was taking tablets with water (as was recommended by the third pictogram - take a tablet with water). Because the use of tablets with water was the most compromised dimension of adherence studied, improvement of this behavior requires additional studies.
In Poland, every package insert of metoprolol PR provides information about the required liquid volume recommended to assure effects (the PR form of metoprolol should be used in the morning, with a minimum half a glass of water, and it cannot be crushed). The pictogram highlighted this information; however, an improvement was not observed, and many patients swallowed medicines without or with not enough liquid.

Non-adherence to recommended therapy can lead to ineffectiveness as well as the manifestation of unintended adverse reactions [13, 14]. Taking a PR formulation requires specific patient behavior and should be considered an important matter for both physicians and pharmacists $[15,16]$. At the first interview over $10 \%$ of patients from both groups crushed the tablets and over $40 \%$ of participants only sometimes swallowed the tablets with water. This observation indicates that information received from physicians and pharmacists may be insufficient for the correct use of medications.

Strategies to improve medication adherence in clinical practice are necessary, given the consequences of medication misuse [6]. Pharmacists, who provide pharmaceutical counseling, play a vital role in this respect [17-19]. The literature indicates many advantages of patient counseling; among others, patients become aware of the importance of therapy, understand doctors' recommendations, become active participants in the treatment process, and thus better follow the treatment recommendations [20].

Patients who received pictograms were able to favorably change their behavior, in contrast to those who were not using pictograms. This is particularly evident with omitting doses, crushing tablets, taking the right dose with the right frequency, and at the correct time of day. Finally, the pictograms were well accepted by the vast majority of participants, which can be understood as a good prognosis for this technology in the future.

Pictograms contribute to a better understanding of the drug's information, especially for the elderly or patients with low health literacy [9]. In our study, the patients' median age was 67 years in the standard pharmacy practice group and 64 years for patients in the pictogram group. Moreover, most of the patients in both groups were taking more than 4 drugs. For this reason, adherence to therapy for these groups of patients is particularly important.

The use of pictograms should be classified as a behavioral technique aimed at changing patient behavior by constantly reminding the patient of proper medication usage. Despite the long history 
of using pictograms, their effectiveness is still a matter of controversy, particularly due to the risk of misunderstanding the pictogram [21-23]. Yin et al. [24] conducted a high-quality randomized study and that showed that pictogram-based intervention decreased the rate of dosing errors among caregivers whose children were treated at an urban pediatric emergency department. Similarly, a study from Malaysia proved the positive role of pictograms in improving the quality of use of oral liquid medicines. Another study suggests that pictograms significantly increased dosing accuracy [25]. The results of a recently published analysis of 771 studies aiming to improve therapeutic adherence showed that healthcare professionals should focus more on behavioral interventions, especially those based on the development of drug use habits, than on cognitive strategies to change the knowledge and beliefs of patients [26].

Although our study only refers to one product (metoprolol), the positive results obtained may be an incentive to extend the use of pictograms to other drugs used in cardiac diseases. Undoubtedly, all pictograms should be designed carefully and tested before their implementation to routine practice [10]. Moreover, pictograms need to be adjusted to local requirements, both formally determined by local summaries of products characteristics and social determinates, which may significantly improve understanding and acceptance levels [19]. For clarity, pictograms should be supplemented by a clear and simple oral statement provided by healthcare professionals [27]. It is worth mentioning that among illiterate populations, verbal explanations are indispensable, and in many clinical scenarios they are crucial to effective comprehension of drug-related information conveyed in pictograms [28]. While our study indicates that pictograms exert a substantial impact on patient behaviors and therapeutic adherence, we were not able to rule out the potential bias related to the study methods. Randomization of pharmacies instead of patients would affect the objectivity of findings. Moreover, the study was conducted in community pharmacies belonging to Farenta Research. These pharmacies, relying on the pharmacy standards, can provide patients with a better quality of care, which may affect the obtained results. The selfreported medication adherence measures used in the study can provide valuable information despite their limitations. Patients may tend to evaluate their adherence better than it really is. Thus, the used questionnaire offered many options to express the level of adherence, but the data analysis was conservative. It was assumed that only total adherence was considered as a positive outcome, and all other options as non-adherence. The change from non-adherence to partial adherence was not considered as an improvement.

\section{Conclusions}

Based on these results, pictograms can be an effective tool dedicated to improving patient adherence. Pictograms were accepted by patients and effectively changed most of their inappropriate behaviors. Our study certainly confirmed that pictograms significantly improve patients' compliance, particularly in the areas of non-omitted drug doses and indications not to crush tablets. Importantly, an improvement in compliance in this respect in the group of patients with standard practice was not observed. Further randomized clinical studies aimed at the evaluation of long-term effectiveness of pictograms are strongly warranted. Further clinical studies should also focus on health care providers' perception of pictograms and how pictograms influence regular work in community pharmacies. This type of research may improve the transition pictograms from research to routine settings.

\section{Acknowledgments}

The authors thanks the personnel of the pharmacies and patients who participated in the study.

\section{Funding}

This work was supported by the National Centre of Research and Development in Warsaw and the LQT Fund S.A. in Gdansk (project Bridge Alfa).

\section{Conflict of interest: None declared}

\section{References}

1. Park LG, Howie-Esquivel J, Chung ML, et al. A text messaging intervention to promote medication adherence for patients with coronary heart disease: a randomized controlled trial. Patient Educ Couns. 2014; 94(2): 261-268, doi: 10.1016/j. pec.2013.10.027, indexed in Pubmed: 24321403.

2. Yap A, Thirumoorthy T, Kwan Y. Medication adherence in the elderly. J Clin Gerontol Geriat. 2016; 7(2): 64-67, doi: 10.1016/j. jcgg.2015.05.001.

3. Kvarnström K, Airaksinen M, Liira H. Barriers and facilitators to medication adherence: a qualitative study with general practitioners. BMJ Open. 2018; 8(1): e015332, doi: 10.1136/bmjopen-2016-015332, indexed in Pubmed: 29362241.

4. Cushing A, Metcalfe R. Optimizing medicines management: From compliance to concordance. Ther Clin Risk Manag. 2007; 3(6): 1047-1058, indexed in Pubmed: 18516274. 
5. Okumura LM, Rotta I, Correr CJ. Assessment of pharmacist-led patient counseling in randomized controlled trials: a systematic review. Int J Clin Pharm. 2014; 36(5): 882-891, doi: 10.1007/ s11096-014-9982-1, indexed in Pubmed: 25052621.

6. Kim S, Shin DW, Yun JM, et al. Medication adherence and the risk of cardiovascular mortality and hospitalization among patients with newly prescribed antihypertensive medications. Hypertension. 2016; 67(3): 506-512, doi: 10.1161/HYPERTENSIONAHA.115.06731, indexed in Pubmed: 26865198.

7. Sletvold H, Sagmo LA, Torheim EA. Impact of pictograms on medication adherence: A systematic literature review. Patient Educ Couns. 2020; 103(6): 1095-1103, doi: 10.1016/j. pec.2019.12.018, indexed in Pubmed: 31924384.

8. Dowse R. Pharmacists, are words enough? The case for pictograms as a valuable communication tool. Res Social Adm Pharm. 2020 [Epub ahead of print], doi: 10.1016/j.sapharm.2020.10.013, indexed in Pubmed: 33139214.

9. Ng AWY, Chan AHS, Ho VWS. Comprehension by older people of medication information with or without supplementary pharmaceutical pictograms. Appl Ergon. 2017; 58: 167-175, doi: 10.1016/j.apergo.2016.06.005, indexed in Pubmed: 27633210.

10. Barros IMC, Alcântara TS, Mesquita AR, et al. The use of pictograms in the health care: a literature review. Res Social Adm Pharm. 2014; 10(5): 704-719, doi: 10.1016/j.sapharm.2013.11.002, indexed in Pubmed: 24332470.

11. Merks P, Świeczkowski D, Balcerzak M, et al. The evaluation of pharmaceutical pictograms among elderly patients in community pharmacy settings: a multicenter pilot study. Patient Prefer Adherence. 2018; 12: 257-266, doi: 10.2147/PPA.S150113, indexed in Pubmed: 29497281.

12. Reichheld FMR. The ultimate question 2.0. Now net promoter companies thrive in a customer-driven world. Harvard Business Review Press, Boston MA 2011.

13. Jankowski P, Czarnecka D, Wolfshaut-Wolak R, et al. Secondary prevention of coronary artery disease in contemporary clinical practice. Cardiol J. 2015; 22(2): 219-226, doi: 10.5603/ CJ.a2014.0066, indexed in Pubmed: 25299500.

14. Kubica A, Obońska K, Kasprzak M, et al. Prediction of high risk of non-adherence to antiplatelet treatment. Kardiol Pol. 2016; 74(1): 61-67, doi: 10.5603/KP.a2015.0117, indexed in Pubmed: 26101025.

15. Swieczkowski D, Merks P, Gruchala M, et al. Pharmaceutical services as a tool to improve outcomes in patients with cardiovascular diseases. Int J Cardiol. 2016; 222(11): 238-241, doi: 10.1016/j.ijcard.2016.07.189, indexed in Pubmed: 27497101.

16. Mogielnicki M, Swieczkowski D, Bachorski W, et al. The Food and Drug Administration (FDA) and the European Medicines Agency (EMA) perspective on cardiovascular Polypill: A multidimensional concept. Cardiol J. 2016; 23(5): 515-517, doi: 10.5603/ CJ.2016.0074, indexed in Pubmed: 27723064.

17. Smith DH, Kramer JM, Perrin N, et al. A randomized trial of direct-to-patient communication to enhance adherence to beta- -blocker therapy following myocardial infarction. Arch Intern Med. 2008; 168(5): 477-483; quiz 447, doi: 10.1001/archinternmed.2007.132, indexed in Pubmed: 18332291.

18. Wong MCS, Liu KQL, Wang HHX, et al. Effectiveness of a pharmacist-led drug counseling on enhancing antihypertensive adherence and blood pressure control: a randomized controlled trial. J Clin Pharmacol. 2013; 53(7): 753-761, doi: 10.1002/ jcph.101, indexed in Pubmed: 23677794.

19. Rosen MI, Rigsby MO, Salahi JT, et al. Electronic monitoring and counseling to improve medication adherence. Behav Res Ther. 2004; 42(4): 409-422, doi: 10.1016/S0005-7967(03)00149-9, indexed in Pubmed: 14998735.

20. Mohiuddin AK. Patient counseling: a complete guide for compliance. J Applied Pharma Sci Res. 2019: 1-10, doi: 10.31069/japsr. v1i4.1.

21. Mansoor LE, Dowse R. Effect of pictograms on readability of patient information materials. Ann Pharmacother. 2003; 37(7-8): 1003-1009, doi: 10.1345/aph.1C449, indexed in Pubmed: 12841808 .

22. Kim H, Nakamura C, Zeng-Treitler Q. Assessment of pictographs developed through a participatory design process using an online survey tool. J Med Internet Res. 2009; 11(1): e5, doi: 10.2196/ jmir.1129, indexed in Pubmed: 19275981.

23. Cloutier M, Vaillancourt R, Pynn D, et al. Design and development of culture-specific pictograms for type 2 diabetes mellitus education and counselling. Can J Diabetes. 2014; 38(6): 379-392, doi: 10.1016/j.jcjd.2014.03.010, indexed in Pubmed: 25028197.

24. Yin HS, Dreyer BP, van Schaick L, et al. Randomized controlled trial of a pictogram-based intervention to reduce liquid medication dosing errors and improve adherence among caregivers of young children. Arch Pediatr Adolesc Med. 2008; 162(9): 814-822, doi: 10.1001/archpedi.162.9.814, indexed in Pubmed: 18762597.

25. Chan HK, Hassali MA, Lim CJ, et al. Improving pediatric liquid medication labeling of the hospital information system in Malaysia: qualitative analysis of pharmacists' perceptions. Pharm Pract (Granada). 2016; 14(2): 699, doi: 10.18549/PharmPract.2016.02.699, indexed in Pubmed: 27382422.

26. Conn VS, Ruppar TM. Medication adherence outcomes of 771 intervention trials: Systematic review and meta-analysis. Prev Med. 2017; 99: 269-276, doi: 10.1016/j.ypmed.2017.03.008, indexed in Pubmed: 28315760.

27. Braich PS, Almeida DR, Hollands S, et al. Effects of pictograms in educating 3 distinct low-literacy populations on the use of postoperative cataract medication. Can J Ophthalmol. 2011; 46(3): 276-281, doi: 10.1016/j.jcjo.2011.05.004, indexed in Pubmed: 21784215.

28. Joshi Y, Kothiyal P. A pilot study to evaluate pharmaceutical pictograms in a multispecialty hospital at dehradun. J Young Pharm. 2011; 3(2): 163-166, doi: 10.4103/0975-1483.80306, indexed in Pubmed: 21731363. 